\title{
Temperature fluctuations in an inhomogeneous diffusive fluid
}

\author{
Z. Haba \\ Institute of Theoretical Physics, University of Wroclaw, \\ 50-204 Wroclaw, Plac Maxa Borna 9, Poland \\ email:zhab@ift.uni.wroc.pl
}

June 14, 2021

\begin{abstract}
We discuss metric perturbations of the relativistic diffusion equation around the homogeneous Jüttner equilibrium of massless particles in a homogenous expanding universe. We describe the perturbations corresponding to the gravitational wave background. We show that the lowest order perturbation can be treated as a variation of temperature. We derive a formula expressing temperature fluctuations in terms of the diffusion and tensor power spectrum. We discuss the multipole expansion of the fluctuations in the presence of diffusion.
\end{abstract}

\section{Introduction}

The currently accepted concordance cosmological model $(\Lambda \mathrm{CDM})[1]$ is in excellent agreement with observational data. However, the incorporation of dark matter and dark energy in this model means that some forms of matter and their interactions remain unknown. We may assume that at present only the gravitational interactions are relevant. However, the unknown particles and their non-gravitational interactions might play a significant role in the early universe. If the early universe is described in terms of known particles and interactions then the lack of information about the remaining forms of matter will be experienced as a non-conservation of energy-momentum of the observed particles. The energy will dissipate into a surrounding of "dark matter" like the energy of Brownian particles does. We suggest the relativistic diffusion as a model of energy dissipation. The diffusion comes from a Markovian (no memory) approximation and does not depend on the details of the interactions. In our earlier paper [2] we have discussed a diffusion of massless particles in a homogeneous expanding metric. The diffusion equation determines a phase-space distribution which defines the energy-momentum of diffusing particles. The diffusion 
equation for massless particles has as a solution the Jüttner distribution with a time-dependent temperature. We have discussed also a non-linear version of the diffusion equation (taking into account the quantum statistics) which leads to the time-dependent Planck distribution. We insert the energy-momentum of diffusing particles into the Einstein equations. It comes out that a compensation of the energy-momentum non-conservation leads to a time-dependent cosmological term. We have shown that the time-dependent Jüttner and Planck distributions give the standard Friedmann equations. We have derived a modified Friedmann equation taking into account the diffusive energy dissipation. For general solutions of the diffusion equation the modification leads to a substantial departure from the Friedmann evolution.

In this letter we report on our study of diffusing particles moving in a nonhomogeneous metric. We are interested in structure formation and temperature fluctuations of a dissipative system of particles. Dissipation is unavoidable in a description of the evolution of density perturbations and temperature fluctuations in a surrounding of an unknown matter and interactions. A dissipation is also necessary in order to achieve a smooth behaviour for a large time. The deterministic (Hamiltonian) systems experience a sensitive dependence on initial conditions. Such a dependence is especially undesirable in cosmological models when the knowledge of initial conditions is not available. In this paper we continue a description of dissipation by a relativistic noise. We consider inhomogeneous metric and discuss a solution of the diffusion equation as a perturbation in the inverse temperature. We show that such a perturbative solution of the inhomogeneous diffusion equation can be treated as a variation of temperature. The solution of the diffusion equation can be considered as a perturbation by diffusion of the well-known perturbative solution of the Liouville-Vlasov equation discussed in the context of the integrated Sachs-Wolfe effect [3][4][5]. In this preliminary study we do not intend to explore the complete system of Einstein equations with diffusing particles. We restrict ourselves to the lowest order approximation of tensor perturbations. In this approximation the tensor perturbations can be identified with gravitational waves. The recent BICEP2 results [6] suggest that in an early universe the perturbations by gravitational waves are of the same order of magnitude as the curvature perturbations leading to structure formation. We show that the diffusion can have a significant impact on temperature fluctuations only if the integrated Sachs-Wolfe effect is numerically relevant. It has not been detected in CMB measurements (it is decreasing at small angular scales and its contribution is more difficult to extract from experimental data). Nevertheless, with the growing precision of measurements the integrated Sachs-Wolfe effect corrected by diffusion could give additional important information about the physical origins of the departure from homogeneity.

The plan of the paper is as follows. In sec.2 we derive the well-known [5][7] perturbative solution of the Liouville-Vlasov equation for massless particles in an inhomogeneous metric in a way which can easily be generalized to the diffusion 
equation. In sec. 3 we discuss the relativistic diffusion equation on a space-time dependent metric. The Jüttner distribution with a time-dependent temperature is a solution of the diffusion equation in a homogeneous metric. We look for a perturbative solution of the diffusion equation in an inhomogeneous metric around the Jüttner distribution. We obtain a solution which can be interpreted as a diffusive variation of temperature in a space-time dependent metric. In sec.4 we calculate correlation functions of temperature fluctuations in a diffusive system which result from tensor perturbations in a quantum vacuum. We discuss the form of the temperature correlations and their multipole expansion which follow from the metric fluctuations. Some examples of metric fluctuations are discussed in the Appendix.

\section{Einstein-Liouville-Vlasov equations}

In this section we solve perturbatively the Einstein-Liouville-Vlasov equation describing a distribution of classical trajectories (see [8][9] for its application in general relativity). We decompose

$$
g_{\mu \nu}=\bar{h}_{\mu \nu}+h_{\mu \nu},
$$

where $\bar{h}_{\mu \nu}$ describes homogenous metric in the conformal time and

$$
d s^{2}=g_{\mu \nu} d x^{\mu} d x^{\nu}=a^{2}\left(d t^{2}-d \mathbf{x}^{2}-\gamma_{i j} d x^{i} d x^{j}\right) .
$$

We decompose the Christoffel symbols corresponding to the perturbation (1)

$$
\Gamma_{\nu \rho}^{\mu}=\bar{\Gamma}_{\nu \rho}^{\mu}+\delta \Gamma_{\nu \rho}^{\mu} .
$$

Using eq.(3) we write the Liouville equation in the form

$$
\left(p^{\mu} \partial_{\mu}^{x}-\bar{\Gamma}_{\mu \nu}^{k} p^{\mu} p^{\nu} \partial_{k}\right) \Omega=\delta \Gamma_{\mu \nu}^{k} p^{\mu} p^{\nu} \partial_{k} \Omega,
$$

where $\partial_{\mu}^{x}=\frac{\partial}{\partial x^{\mu}}$ and $x=(t, \mathbf{x})$ (boldface letters denoting the three vectors), $\partial_{k}=\frac{\partial}{\partial p^{k}}$ denotes derivatives over momenta.

For massless particles and in the homogeneous metric $\left(h_{\mu \nu}=0\right)$ any function $F\left(a^{2}|\mathbf{p}|\right)$ is the solution of eq.(4)[10]. We are interested in classical equilibrium states described by the Jüttner distribution [11]

$$
\Omega_{E}=\exp \left(-a^{2} \beta|\mathbf{p}|\right)
$$

where $\beta \equiv \frac{1}{T}$ is the inverse temperature and

$$
\mathbf{p}^{2}=\sum_{j} p^{j} p^{j}
$$


In an inhomogeneous metric (2) we consider the Jüttner distribution of the form

$$
\Omega_{E}^{g}=\exp \left(-\beta p_{0}\right),
$$

where (from $\left.g_{\mu \nu} p^{\mu} p^{\nu}=0\right)$

$$
p_{0}=g_{00} p^{0}=a^{2}\left(\mathbf{p}^{2}+p^{j} \gamma_{j k} p^{k}\right)^{\frac{1}{2}} .
$$

Then, the solution of eq.(4) (neglecting second order terms in $\gamma$ ) can be expressed as

$$
\Omega=\Omega_{E}^{g}+\beta p_{0} \Theta \Omega_{E}^{g},
$$

where $n^{k}=p^{k}|\mathbf{p}|^{-1}$ and $\Theta$ is the solution of the equation

$$
\partial_{t} \Theta+n^{k} \partial_{k}^{x} \Theta=-\frac{1}{2} n^{j} n^{k} \partial_{t} \gamma_{j k} .
$$

It follows that (till the first order in $\delta T$ )

$$
\Omega=\exp \left(-\frac{p_{0}}{T+\delta T}\right)
$$

where

$$
\delta T=T \Theta
$$

Eq.(9) has the solution

$$
\Theta(t, \mathbf{x})=\Theta_{0}(\mathbf{x}-\mathbf{n} t)-\frac{1}{2} \int_{0}^{t} \partial_{s} \gamma_{j k}(s, \mathbf{x}-(t-s) \mathbf{n}) n^{j} n^{k} d s
$$

with the initial condition $\Theta_{0}(\mathbf{x})$. For a realistic system of a gas of interacting particles the collision terms should be taken into account. A solution of the Boltzmann equation (with collision terms) for the temperature variation in an inhomogeneous metric has been developed by means of numerical methods [12][13] (CMBFast and CAMB, see also the discussion in [14]). In analytic methods some approximations to the collision term are applied . Dodelson ( sec.4.4 in [7]) derives a friction term as the relaxation time approximation to the Boltzmann equation (see [15] for a discussion of such a term in a general metric). In the next section we consider diffusion approximations which can simulate interactions with the dark matter (a diffusion approximation for the collision term in the relativistic Boltzmann equation has been discussed in [16]). The diffusion and friction relax the dependence on the initial conditions (in the sense that the initial condition is irrelevant for the large time behaviour).

\section{Relativistic diffusion}

In this section we first review the theory of the relativistic diffusion. Then, we solve the diffusion equation in a space-time dependent metric in a close analogy 
to the solution of the Liouville equation in sec.2. We look for a relativistic generalization of the Kramers diffusion defined on the phase space. It is determined in the unique way by the requirement that the diffusing particle moves on the mass-shell $\mathcal{H}_{+}($see $[17][18][19][20][21])$

$$
g_{\mu \nu} p^{\mu} p^{\nu}=m^{2} .
$$

The diffusion is generated by the Laplace-Beltrami operator $\triangle_{H}^{m}$ on $\mathcal{H}_{+}$

$$
\triangle_{H}^{m}=\frac{1}{\sqrt{G}} \partial_{j} G^{j k} \sqrt{G} \partial_{k},
$$

where

$$
G^{j k}=m^{2} g^{j k}+p^{j} p^{k},
$$

$\partial_{j}=\frac{\partial}{\partial p^{j}}$ and $G=\operatorname{det}\left(G_{j k}\right)$ is the determinant of $G_{j k}$.

The transport equation for the linear diffusion generated by $\triangle_{H}$ reads

$$
\left(p^{\mu} \partial_{\mu}^{x}-\Gamma_{\mu \nu}^{k} p^{\mu} p^{\nu} \partial_{k}\right) \Omega=\kappa^{2} \triangle_{H}^{m} \Omega,
$$

where $\kappa^{2}$ is the diffusion constant. Eq.(16) is a generalization of the Liouville equation (4) incorporating dissipation.

Next, we consider a diffusion at finite temperature $\beta^{-1}[19][20][21][22][23]$ (in a frame moving with the heat bath; this equation can be considered as a linear version of the Kompaneets equation [24][25])

$$
\left(p^{\mu} \partial_{\mu}^{x}-\Gamma_{\mu \nu}^{k} p^{\mu} p^{\nu} \partial_{k}\right) \Omega=\kappa^{2} p_{0} \partial_{j}\left(G^{j k} p_{0}^{-1} \partial_{k}+\beta p^{j}\right) \Omega .
$$

Here, $p_{0}$ is determined from eq.(13). $\Omega_{E}$ (5) is the solution of eq.(17) in the homogeneous metric in the massless (ultrarelativistic) limit $m=0$. In our interpretation the expanding dark matter plays the role of a heat bath. The general solution of eq.(17) tends to $\Omega_{E}$ for a large time.

In the inhomogeneous metric (2) we write the solution in the form (10)

$$
\Omega=\Omega_{E}^{g}+\beta p_{0} \Theta \Omega_{E}^{g}+\beta r a^{2} \Omega_{E}^{g}=\left(1+\beta r a^{2}\right) \exp \left(-\frac{p_{0}}{T+T \Theta}\right)=\exp \left(-\frac{p_{0}-a^{2} r}{T+T \Theta}\right)
$$

where the equality is up to the second order terms in $\Theta$ and $r$. It can be seen from eq.(18) that the term $-a^{2} r$ has the meaning of a supplementary gravitational potential induced by the diffusion in a heat bath. Inserting this formula in eq.(17) we obtain equations for the temperature fluctuation $\Theta$ and for $r$

$$
\begin{gathered}
\partial_{t} \Theta+n^{k} \partial_{k}^{x} \Theta+\kappa^{2} \beta a^{2} \Theta=-\frac{1}{2} n^{j} n^{k} \partial_{t} \gamma_{j k}, \\
\partial_{t} r+n^{k} \partial_{k}^{x} r+2 \mathcal{H} r=3 \kappa^{2} \Theta
\end{gathered}
$$

where

$$
n^{k}=p^{k}|\mathbf{p}|^{-1} .
$$


The solution of eq.(19) reads

$$
\begin{aligned}
& \Theta_{t}(\mathbf{x})=\exp \left(-\beta \kappa^{2} \int_{0}^{t} a^{2}(s) d s\right) \Theta_{0}(\mathbf{x}-t \mathbf{n}) \\
& -\frac{1}{2} \int_{0}^{t} d s \exp \left(-\beta \kappa^{2} \int_{s}^{t} a^{2}(r) d r\right) \partial_{s} \gamma_{j k} n^{j} n^{k}(s, \mathbf{x}-(t-s) \mathbf{n}),
\end{aligned}
$$

where $\Theta_{0}$ is the initial condition. Note that in cosmological models (for a large $t$ the scale factor $a$ is increasing) the term depending on the initial condition disappears. Eq.(22) describes a variation of the temperature of a gas of particles interacting with an environment. We have approximated the interaction by diffusion.

\section{Temperature fluctuations}

The aim of this section is to obtain explicit analytic expressions for temperature fluctuations. We restrict ourselves to tensor perturbations [26][27](the general perturbations require a solution of Einstein equations with an energymomentum tensor describing matter distribution [28][29]). The formula for the correlations of temperature fluctuations follows from eq.(22) and the discussion in Appendix (we neglect the term depending on the initial condition $\Theta_{0}$ which disappears at large time)

$$
\begin{aligned}
& \left\langle\Theta(t, \mathbf{n}) \Theta\left(t, \mathbf{n}^{\prime}\right)\right\rangle= \\
& =\frac{1}{4}(2 \pi)^{-3} \int_{0}^{t} d s \int_{0}^{t} d s^{\prime} \int d \mathbf{q} F\left(s, s^{\prime}, q\right) \exp \left(-\beta \kappa^{2}\left(\int_{s}^{t}+\int_{s^{\prime}}^{t}\right) d r a^{2}(r)\right) \\
& \left(2\left(\mathbf{n} \Delta(\mathbf{q}) \mathbf{n}^{\prime}\right)^{2}-(\mathbf{n} \Delta(\mathbf{q}) \mathbf{n})\left(\mathbf{n}^{\prime} \Delta(\mathbf{q}) \mathbf{n}^{\prime}\right)\right) \exp \left(-i(t-s) \mathbf{n q}+i\left(t-s^{\prime}\right) \mathbf{n}^{\prime} \mathbf{q}\right)
\end{aligned}
$$

where

$$
\begin{gathered}
\mathbf{n} \Delta(\mathbf{q}) \mathbf{n}^{\prime}=\mathbf{n} \mathbf{n}^{\prime}-\mathbf{q}^{-2}(\mathbf{q n})\left(\mathbf{q} \mathbf{n}^{\prime}\right) \equiv \Delta\left(\mathbf{n} \mathbf{n}^{\prime}, \mathbf{e n}, \mathbf{e n}^{\prime}\right) \\
\mathbf{n} \Delta(\mathbf{q}) \mathbf{n}=1-\mathbf{q}^{-2}(\mathbf{q} \mathbf{n})^{2} \equiv \delta(\mathbf{e n})
\end{gathered}
$$

and we write $\mathbf{q}=q \mathbf{e}$ with a unit vector $\mathbf{e}$. In eq.(23)

$$
F\left(s, s^{\prime}, q\right)=\partial_{s} \partial_{s^{\prime}} P\left(s, s^{\prime}, q\right)
$$

where $P$ is the correlation function (eqs.(2)-(4) in the Appendix) of tensor perturbations. $F(s, s, q)$ is the power spectrum of the gravitational waves (eq.(7) of the Appendix). The various factors in the formula (23) for temperature fluctuations result from general rules: the assumption that the tensor perturbations are transverse and traceless and the power spectrum $F$ describes the density of Fourier modes. The extra damping factor $\exp \left(-\beta \kappa^{2} \int a^{2}\right)$ comes from the friction leading to the equilibrium in eq.(17) (a consequence of the detailed balance). The expression (23) for temperature fluctuations can be finite for $t \rightarrow \infty$ owing to the damping exponential factor (even if the corresponding expression without the diffusion grows to infinity). As pointed out in the Appendix the power spectrum $F(s, s, q)$ can be associated with the energy of gravitational 
waves. By means of the temperature fluctuations (23) $F$ is accessible to measurements. We discuss here some consequences of various forms of $F$.

At the initial stage of our study of eq.(23) we follow the monograph by Mukhanov [5]. In the integral $d \mathbf{q}=d q q^{2} d \mathbf{e}$ we integrate first over $\mathbf{e}$ in the exponential in eq.(23). We obtain

$$
\begin{aligned}
& \int d \mathbf{e} \exp \left(-i(t-s) \mathbf{n q}+i\left(t-s^{\prime}\right) \mathbf{n}^{\prime} \mathbf{q}\right) \\
& =2 \pi q^{-1}\left|(t-s) \mathbf{n}-\left(t-s^{\prime}\right) \mathbf{n}^{\prime}\right|^{-1} \sin \left(\left|(t-s) q \mathbf{n}-\left(t-s^{\prime}\right) q \mathbf{n}^{\prime}\right|\right) .
\end{aligned}
$$

Next, we use the expansion

$$
\begin{aligned}
& q^{-1}\left|(t-s) \mathbf{n}-\left(t-s^{\prime}\right) \mathbf{n}^{\prime}\right|^{-1} \sin \left(\left|(t-s) q \mathbf{n}-\left(t-s^{\prime}\right) q \mathbf{n}^{\prime}\right|\right) \\
& =\sum_{l=0}^{\infty}(2 l+1) j_{l}(q(t-s)) j_{l}\left(q\left(t-s^{\prime}\right)\right) P_{l}\left(\mathbf{n} \mathbf{n}^{\prime}\right) .
\end{aligned}
$$

$j_{l}$ is the Bessel spherical function related to the Bessel function $J$ [35]

$$
j_{l}(z)=\sqrt{\frac{\pi}{2 z}} J_{l+\frac{1}{2}}(z)
$$

and $P_{l}$ are the Legendre polynomials.

If $F$ is known then there remains to perform the integrals over $s$ and $q$ using eqs.(23) and (28) in order to obtain

$$
\left\langle\Theta(t, \mathbf{n}) \Theta\left(t, \mathbf{n}^{\prime}\right)\right\rangle=\sum_{l=0}^{\infty}(2 l+1) \tilde{D}_{l}\left(t, \mathbf{n n}^{\prime}\right) P_{l}\left(\mathbf{n n}^{\prime}\right)=\sum_{l=0}^{\infty}(2 l+1) C_{l}(t) P_{l}\left(\mathbf{n n}^{\prime}\right),
$$

In eq.(29) $\tilde{D}_{l} P_{l}$ still must be expanded in Legendre polynomials if the coefficients $C_{l}$ are to be independent of the angle. We have from eqs.(23)-(28)

$$
\begin{aligned}
& \tilde{D}_{l}=\frac{1}{16 \pi^{2}} \int_{0}^{t} d s \int_{0}^{t} d s^{\prime} \int d q q^{2} F\left(s, s^{\prime}, q\right) \exp \left(-\beta \kappa^{2}\left(\int_{s}^{t}+\int_{s^{\prime}}^{t}\right) d r a^{2}(r)\right) \\
& \left(2 \Delta\left(\mathbf{n n}^{\prime},-i \partial_{s}, i \partial_{s^{\prime}}\right)^{2}-\delta\left(-i \partial_{s}\right) \delta\left(i \partial_{s^{\prime}}\right)\right) j_{l}(q(t-s)) j_{l}\left(q\left(t-s^{\prime}\right)\right) .
\end{aligned}
$$

Let us consider only the first term in eq.(30) (denoted $D_{l}$ ) resulting from the expansion

$$
2 \Delta\left(\mathbf{n n}^{\prime},-i \partial_{s}, i \partial_{s^{\prime}}\right)^{2}-\delta\left(-i \partial_{s}\right) \delta\left(i \partial_{s^{\prime}}\right)=2\left(\mathbf{n n}^{\prime}\right)^{2}-1+O\left(\partial_{s}, \partial_{s^{\prime}}\right)
$$

where $O$ is a polynomial of at least first order in derivatives. The remaining terms in eq.(30) can easily be calculated by taking derivatives $\partial_{s}$ of $D_{l}$ ( the method to express the remaining terms by time derivatives of $D_{l}$ is taken from [5]). We have

$$
\begin{aligned}
& D_{l}=\frac{1}{16 \pi^{2}}\left(2\left(\mathbf{n n}^{\prime}\right)^{2}-1\right) \int_{0}^{t} d s^{\prime} \int_{0}^{t} d s \exp \left(-\beta \kappa^{2}\left(\int_{s}^{t}+\int_{s^{\prime}}^{t}\right) d r a^{2}(r)\right) \\
& \int_{0}^{\infty} d q q^{2} F\left(s, s^{\prime}, q\right) j_{l}(q(t-s)) j_{l}\left(q\left(t-s^{\prime}\right)\right)
\end{aligned}
$$

$D_{l}$ as well as $\tilde{D}_{l}$ have the same behaviour at large $l$ as $C_{l}$ in eq.(29). We make a simplifying assumption

$$
F\left(s, s^{\prime}, q\right)=f\left(s, s^{\prime}\right) \sigma(q)
$$


First, we estimate $D_{l}$ for a large $l$. According to the Limber asymptotic formula [30][31] (see also the calculations in sec.9 of [32])

$$
\begin{aligned}
& D_{l}=\frac{1}{32 \pi}\left(2\left(\mathbf{n n}^{\prime}\right)^{2}-1\right) \int_{0}^{t} d s(t-s)^{-2} \\
& f(s, s) \exp \left(-2 \beta \kappa^{2} \int_{s}^{t} d r a^{2}(r)\right) \sigma\left(\frac{l+\frac{1}{2}}{t-s}\right)\left(1+O\left(l^{-2}\right)\right) .
\end{aligned}
$$

Here, $O\left(l^{-2}\right)$ denotes terms of order $l^{-2}$. For the inflationary almost flat spectrum (small $\epsilon$ )

$$
\sigma(q)=A q^{-3+\epsilon}
$$

Eq.(34) can also apply to the model describing the appearance of inflation after the equilibrium phase [33]. Then, $\epsilon \rightarrow-1+\epsilon$ in eq.(34). For any $\epsilon<2$ in the power spectrum (34) we get from eq.(33)the asymptotic behaviour

$$
\begin{aligned}
& D_{l}=A\left(l+\frac{1}{2}\right)^{-3+\epsilon} \frac{1}{32 \pi}\left(2\left(\mathbf{n n}^{\prime}\right)^{2}-1\right) \int_{0}^{t} d s(t-s)^{1-\epsilon} \\
& f(s, s) \exp \left(-2 \beta \kappa^{2} \int_{s}^{t} d r a^{2}(r)\right)\left(1+O\left(l^{-2}\right)\right) .
\end{aligned}
$$

As can be seen from eq.(35) the asymptotics in $l$ does not depend on the diffusion. We can derive an exact result for the integral (31) using the formula 6.574 of $[35](\gamma<\sigma)$

$$
\begin{aligned}
& \int_{0}^{\infty} d q q^{-2+\epsilon} J_{l+\frac{1}{2}}(\sigma q) J_{l+\frac{1}{2}}(\gamma q)=\frac{1}{2^{2-\epsilon}} \frac{\Gamma\left(l+\frac{\epsilon}{2}\right)}{\Gamma\left(\frac{3}{2}-\frac{\epsilon}{2}\right) \Gamma\left(l+\frac{3}{2}\right)}\left(\frac{\gamma}{\sigma}\right)^{l} \sqrt{\gamma \sigma} \sigma^{\epsilon} \\
& F\left(l+\frac{\epsilon}{2},-\frac{1}{2}+\frac{\epsilon}{2}, l+\frac{3}{2}, \frac{\gamma^{2}}{\sigma^{2}}\right),
\end{aligned}
$$

where $F(\alpha, \beta, \gamma, z)$ denotes the hypergeometric function. Applying eq.(36) we obtain

$$
\begin{aligned}
& D_{l}(t)=\frac{1}{16 \pi} A\left(2\left(\mathbf{n n}^{\prime}\right)^{2}-1\right) \frac{1}{2^{2-\epsilon}} \frac{\Gamma\left(l+\frac{\epsilon}{2}\right)}{\Gamma\left(\frac{3}{2}-\frac{\epsilon}{2}\right) \Gamma\left(l+\frac{3}{2}\right)} \int_{0}^{t} d s \int_{0}^{s} d s^{\prime}\left(\frac{t-s^{\prime}}{t-s}\right)^{l}(t-s)^{\epsilon} \\
& f\left(s, s^{\prime}\right) \exp \left(-\beta \kappa^{2}\left(\int_{s}^{t}+\int_{s^{\prime}}^{t}\right) d r a^{2}(r)\right) F\left(l+\frac{\epsilon}{2},-\frac{1}{2}+\frac{\epsilon}{2}, l+\frac{3}{2}, \frac{\left(t-s^{\prime}\right)^{2}}{(t-s)^{2}} .\right.
\end{aligned}
$$

The integral (31) can easily be calculated if $F(32)$ is concentrated at $s=$ $s^{\prime}=s_{d}$. This case describes an instantaneous metric perturbation (the metric perturbation is limited to the moment $s_{d}$ ) corresponding to a sudden decoupling at $s=s_{d}$ from the last scattering surface [5][32]. In such a case we set

$$
f\left(s, s^{\prime}\right)=f_{d} \delta\left(s-s_{d}\right) \delta\left(s^{\prime}-s_{d}\right) .
$$

Hence, $s=s^{\prime}=s_{d}$ in the argument of the hypergeometric function (36). The metric perturbation at a fixed time is applied in the ordinary Sachs-Wolfe effect [3] . In order to relate the integral (31) to the well-known result let us calculate it at $s=s^{\prime}$ and $\epsilon=0$ (Harrison-Zeldovich spectrum) in eq.(36). Then, we can obtain the value of the hypergeometric function at 1 using the formula

$$
F(\alpha, \beta, \gamma, 1)=(F(\alpha,-\beta, \gamma-\beta, 1))^{-1}
$$


and

$$
F(\alpha, \beta, \gamma, 1)=\frac{\Gamma(\gamma) \Gamma(\gamma-\alpha-\beta)}{\Gamma(\gamma-\alpha) \Gamma(\gamma-\beta)} .
$$

The approximation $\gamma \simeq \sigma=t-s_{d}$ in eq.(37) leads to the result

$$
\int_{0}^{\infty} d q q^{-2} J_{l+\frac{1}{2}}(\gamma q) J_{l+\frac{1}{2}}(\gamma q)=\frac{\gamma}{\pi} \frac{(l-1) !}{(l+1) !} .
$$

As a consequence

$$
D_{l}(t)=A f_{d}\left(2\left(\mathbf{n n}^{\prime}\right)^{2}-1\right) \frac{1}{16 \pi}(l(l+1))^{-1} \exp \left(-2 \beta \kappa^{2} \int_{s_{d}}^{t} d r a^{2}(r)\right) .
$$

Hence, if the metric perturbation is applied only at a fixed time then the diffusion gives just a constant factor in the standard formula for the temperature fluctuations [5][32]. This behaviour can change when we admit power spectra which are not of the power-like form . As an example (which can be related to the Gibbs damping of the high energy modes) we consider the metric fluctuations of the form

$$
F\left(s, s^{\prime}, q\right)=Q q^{n-1} \exp (-\beta q) f\left(s, s^{\prime}\right) .
$$

For the power spectrum (40) we have the Limber formula

$$
\begin{aligned}
& D_{l}(t)=\left(l+\frac{1}{2}\right)^{n-1} \frac{1}{32 \pi} Q\left(2\left(\mathbf{n n}^{\prime}\right)^{2}-1\right) \\
& \int_{0}^{t} d s f(s, s)(t-s)^{-1-n} \exp \left(-\frac{\beta\left(l+\frac{1}{2}\right)}{t-s}-\beta \kappa^{2} \int_{s}^{t} d r a^{2}(r)\right)
\end{aligned}
$$

The integral (41) could be calculated by means of the saddle point method. The saddle point $s_{c}$ is determined from the equation

$$
-\frac{1+n}{t-s_{c}}+\beta \frac{l+\frac{1}{2}}{\left(t-s_{c}\right)^{2}}=\beta \kappa^{2} a^{2}\left(s_{c}\right)
$$

In eqs.(41)-(42) the dependence of $D_{l}$ on $l$ is a function of the expansion scale factor $a$ and the dissipation rate $\beta \kappa^{2}$. Note that if $\kappa=0$ then from eq.(41) we obtain $D_{l} \simeq l^{-1}$.

In order to derive an exact formula for the power spectrum (40) we can use the integral 6.612 of [35]

$$
\begin{aligned}
& \int_{0}^{\infty} d q q^{n} \exp (-\lambda q) J_{l+\frac{1}{2}}(\sigma q) J_{l+\frac{1}{2}}(\gamma q)=\sqrt{\gamma \sigma}(-1)^{n} \frac{l !}{\Gamma\left(l+\frac{3}{2}\right)}(\gamma \sigma)^{l} \\
& \partial_{\lambda}^{n}\left(\lambda^{2}+\gamma^{2}+\sigma^{2}\right)^{-l-1} F\left(\frac{1}{2} l+1, \frac{1}{2} l+\frac{1}{2}, l+\frac{3}{2}, \frac{4 \gamma^{2} \sigma^{2}}{\left(\lambda^{2}+\gamma^{2}+\sigma^{2}\right)^{2}}\right) .
\end{aligned}
$$

The formula (43) can be applied for the calculation of $D_{l}$ by means of an expansion of the hypergeometric function in a power series because $\lambda>0$ makes the series quickly convergent. Then,

$$
\begin{aligned}
& D_{l}(t)=\frac{1}{32 \pi}(-1)^{n} Q\left(2\left(\mathbf{n n}^{\prime}\right)^{2}-1\right) \int_{0}^{t} d s^{\prime} \int_{0}^{s^{\prime}} d s\left(\frac{t-s^{\prime}}{t-s}\right)^{l} \\
& \exp \left(-\beta \kappa^{2}\left(\int_{s}^{t}+\int_{s^{\prime}}^{t}\right) d r a^{2}(r)\right) \partial_{\lambda}^{n} F\left(\frac{1}{2} l+1, \frac{1}{2} l+\frac{1}{2}, l+\frac{3}{2}, \frac{4 \gamma^{2} \sigma^{2}}{\left(\lambda^{2}+\gamma^{2}+\sigma^{2}\right)^{2}}\right),
\end{aligned}
$$


where $\gamma=t-s$ and $\sigma=t-s^{\prime}$. The analytic expressions derived in this section give an explicit large $l$ behaviour. The integral formulae for fluctuations could be applied for numerical integration. In comparison with the general Boltzmann equations [12][13] our expressions are rather elementary because we replace the complex collision integrals by the relativistic diffusion.

\section{Discussion and Outlook}

We have found solutions of the relativistic diffusion equation which can be considered as a diffusive disturbance of the standard Liouville equation in a perturbed metric. The diffusion can approximate the collision term in the Boltzmann equation when an interaction with the dark matter is taken into account. The solutions describe temperature variation $\Theta$ in an inhomogeneous metric. In order to determine the temperature variation unambiguously for general perturbations we would need to solve the Einstein equations with the energy-momentum tensor defined by temperature fluctuations. The energymomentum is determined by $\Theta$ and is of the form $\simeq(T+T \Theta)^{4}$. In this paper we restrict ourselves to tensor perturbations which in the lowest order (i.e. , with no energy-momentum on the rhs of Einstein equations) are identical with gravitational waves. Their quantization leads to temperature fluctuations. The recent BICEP2 results indicate that such temperature fluctuations are of the same order of magnitude as the fluctuations resulting from scalar perturbations. Our results for temperature variation reduce to the ones derived earlier by other authors in the absence of diffusion. In the sudden decoupling approximation (ordinary Sachs-Wolfe effect), confirmed by observations within the present experimental accuracy, the dissipation gives only a multiplicative constant. However, in the general formula for the integrated Sachs-Wolfe effect the diffusion contributes along the whole particle's path. It can give an $l$-dependent modification to the multipole expansion coefficients $C_{l}$. The integrated Sachs-Wolfe effect is negligible at large $l$ in comparison with the ordinary Sachs-Wolfe effect. At the present precision of measurements it seems unlikely that the effect of diffusion on tensor perturbations can be measurable. Nevertheless, a determination of the contribution of diffusion may suggest future measurements. We intend to continue the study of the impact of diffusion upon remaining perturbations of the metric (especially scalar perturbations leading to acoustic oscillations). For this purpose we must investigate Einstein equations for the perturbations.

\section{Acknowledgement}

The research is supported by NCN grant DEC-2013/09/B/ST2/03455 


\section{Appendix}

In general, we should insert the energy-momentum tensor $T^{\mu \nu}$ determined by the diffusion (17) into the Einstein equations in order to derive the solution for the metric perturbations $h_{\mu \nu}=a^{2} \gamma_{\mu \nu}$. The solution depends on the linear gravitational waves which are the solutions of Einstein equations at the zeroth order of perturbation corresponding to $T^{\mu \nu}=0$. Inserting the solutions $\gamma_{j k}$ in $\Theta(22)$ we calculate the temperature fluctuations resulting from gravitational waves in the lowest order of perturbation.

We expand solutions of Einstein equations in an external homogeneous metric in plane waves

$$
\gamma_{j k}(t, \mathbf{x})=(2 \pi)^{-\frac{3}{2}} \sum_{\lambda} \int d \mathbf{q}|\mathbf{q}|^{-\frac{1}{2}}\left(a(\lambda, \mathbf{q}) e_{j k}(\lambda, \mathbf{q}) \gamma(t, \mathbf{q}, \lambda) \exp (i \mathbf{q x})+c c\right),
$$

where $c c$ denotes the complex conjugation of the preceding term, $\lambda$ is the polarization of gravitons, $e_{j k}$ are the polarization tensors and $\gamma$ are solutions of the wave equations ( see [36]). Quantizing the Fourier modes we obtain the vacuum correlation functions of gravitons in the transverse-traceless gauge. So, in the momentum space

$$
\left\langle\gamma_{j n}(t, \mathbf{k}) \gamma_{l r}\left(t^{\prime}, \mathbf{q}\right)\right\rangle=\delta(\mathbf{k}+\mathbf{q})(2 \pi)^{-3} P\left(t, t^{\prime}, q\right)\left(\Delta_{j l} \Delta_{r n}+\Delta_{j r} \Delta_{n l}-\Delta_{j n} \Delta_{l r}\right),
$$

where

$$
\Delta_{j l}=\delta_{j l}-q_{j} q_{l} \mathbf{q}^{-2}
$$

and

$$
P\left(t, t^{\prime}, q\right)=q^{-1} \sum_{\lambda, k, j} \overline{e_{j}^{k}(\lambda, \mathbf{q}) \gamma(t, \mathbf{q}, \lambda)} e_{j}^{k}(\lambda, \mathbf{q}) \gamma\left(t^{\prime}, \mathbf{q}, \lambda\right)
$$

$P$ depends only on $q=|\mathbf{q}|$ because of the rotational invariance. Taking the time derivatives in eq.(2)

$\left\langle\partial_{t} \gamma_{j n}(t, \mathbf{k}) \partial_{t^{\prime}} \gamma_{l r}\left(t^{\prime}, \mathbf{q}^{\prime}\right)\right\rangle=\delta(\mathbf{k}+\mathbf{q})(2 \pi)^{-3} F\left(t, t^{\prime}, \mathbf{q}\right)\left(\Delta_{j l} \Delta_{r n}+\Delta_{j r} \Delta_{n l}-\Delta_{j n} \Delta_{l r}\right)$,

where

$$
F\left(t, t^{\prime}, \mathbf{q}\right)=\partial_{t} \partial_{t^{\prime}} P\left(t, t^{\prime}, \mathbf{q}\right)
$$

The power spectrum $F(t, t, q)$ is related to the energy $\epsilon_{g}$ of gravitational waves [34]

$$
\epsilon_{g}=\frac{a^{2}}{32 \pi G} \int d \mathbf{q} F(t, t, q)
$$

We can understand the power spectrum appearing in temperature fluctuations in a wider sense as the energy impulse coming from gravitational waves. We could obtain such generalized $F$ calculating the correlation functions of $\gamma$ in quantum states more general than just the vacuum states. In such a case the impulse described by $F$ can be confined in time. 
As a standard example leading to the power spectrum (34) with $\epsilon=0$ we consider de Sitter space with the metric $d s^{2}=t^{-2}\left(d t^{2}-d \mathbf{x}^{2}\right)$. The correlation functions in the Bunch-Davis vacuum are

$$
P_{d S}\left(t, t^{\prime}, \mathbf{q}\right)=\frac{1}{2|\mathbf{q}|}\left(1+\frac{i\left(t-t^{\prime}\right)}{|\mathbf{q}| t t^{\prime}}+\left(\mathbf{q}^{2} t t^{\prime}\right)^{-1}\right) \exp \left(-i\left(t-t^{\prime}\right)|\mathbf{q}|\right)
$$

For a small $q$

$$
F(t, t, q)=\frac{1}{2} q^{-3} t^{-4}
$$

\section{References}

[1] D. S. Gorbunov and V. A. Rubakov, Introduction to the Theory of the Early Universe. Hot Big Bang Theory,World Scientific, Singapore, 2011

[2] Z. Haba, Class.Quant.Grav.31,075011(2014)

[3] R. Sachs and A. Wolfe, Astroph.J.147,73(1967)

[4] A. J. Nishizawa, arxiv:1404.5102[astro-ph.CO]

[5] V. Mukhanov, Physical Foundations of Cosmology, Cambridge Univ.Press,2005

[6] P.A.R. Ade, et al arXiv:1403.3985[astro-ph.CO]

[7] S. Dodelson, Modern Cosmology, Academic Press, New York, 2003

[8] D. Bancel and Y. Choquet-Bruhat, Commun.Math.Phys.33,83(1973)

[9] H. Andreasson, Living Rev.Relativity, 14,4(2011);arXiv:1106.1367[gr-qc]

[10] J. Bernstein, Kinetic Theory in an Expanding Universe, Cambridge,1988

[11] F. Jüttner, Ann.Phys.(Leipzig)34,856(1911)

[12] U. Seljak and M. Zaldariagga, Astroph J. 469,437(1996)

[13] A. Lewis, A. Challinor and A. Lasemby, Astroph.J. 538 473(2000)

[14] W. Hu and T. Okamoto, arXiv:03080049[astro-ph.CO]

[15] G. M. Kremer, Int.J. Geom.Methods Mod.Phys.11,140006(2014)

[16] G. Chacon-Acosta and G.M.Kremer, Phys.Rev.E76,021201(2007)

[17] G.Schay,PhD thesis,Princeton University,1961 
[18] R.Dudley, Arkiv for Matematik,6,241(1965)

[19] Z. Haba, Phys.Rev.E79,021128(2009)

[20] Z. Haba, Class. Quantum Grav.27,095021(2010)

[21] J.A. Alcantara and S.Calogero, Kin.Rel. Mod.4,401(2011)

[22] J. Dunkel and P. Hänggi, Phys.Rev.E72,036106(2005)

[23] C. Chevalier and F. Debbasch, J.Math.Phys.49,043303(2008)

[24] A.S.Kompaneets, Sov.Phys.JETP 4,730(1957)

[25] Z. Haba, PhysicaA390,2776(2011)

[26] A. Starobinskii, JETP Lett.30,683(1979)

[27] V. Rubakov, M. Sazhin and A. Veryaskin, Phys. Lett.117B,175(1982)

[28] V.F. Mukhanov, H.A. Feldman and R.H.Branderberger, Phys.Rep.215,203(1992)

[29] J. Bardeen, Phys.Rev.D22,1882(1980)

[30] D.N. Limber, Astroph.J.117, 134(1953)

[31] M. LoVerde and N. Afshordi, Phys. Rev.D78,123506(2008)

[32] D.S. Gorbunov and V.A. Rubakov, Introduction to the Theory of the Early Universe. Cosmological Perturbations and Inflationary Theory, World Scientific, 2011

[33] K. Bhattacharya, S. Mohanty and A. Nautiyal, Phys. Rev.Lett.97,251301(2006)

[34] C.W.Misner, K.S. Thorne and J.A. Wheeler, Gravitation, W.H. Freeman Co.,San Francisco,1973

[35] Y.S. Gradshtein and Y.M. Ryzhik, Tables of Integrals, Sums, Series and Products,Nauka, Moscow, 1971 (in Russian)

[36] M. Giovannini, arXiv:1405.630[astro-ph.CO] 